Document downloaded from:

http://hdl.handle.net/10251/95451

This paper must be cited as:

Ortiz Serna, MP.; Díaz Calleja, R.; Sanchis Sánchez, MJ. (2012). Evaluation of Natural Rubber Specific Heat Capacity at High Pressures from DSC Experimental Data at Atmospheric Pressure. Journal of Applied Polymer Science. 128(4):2269-2272. doi:10.1002/app.38118

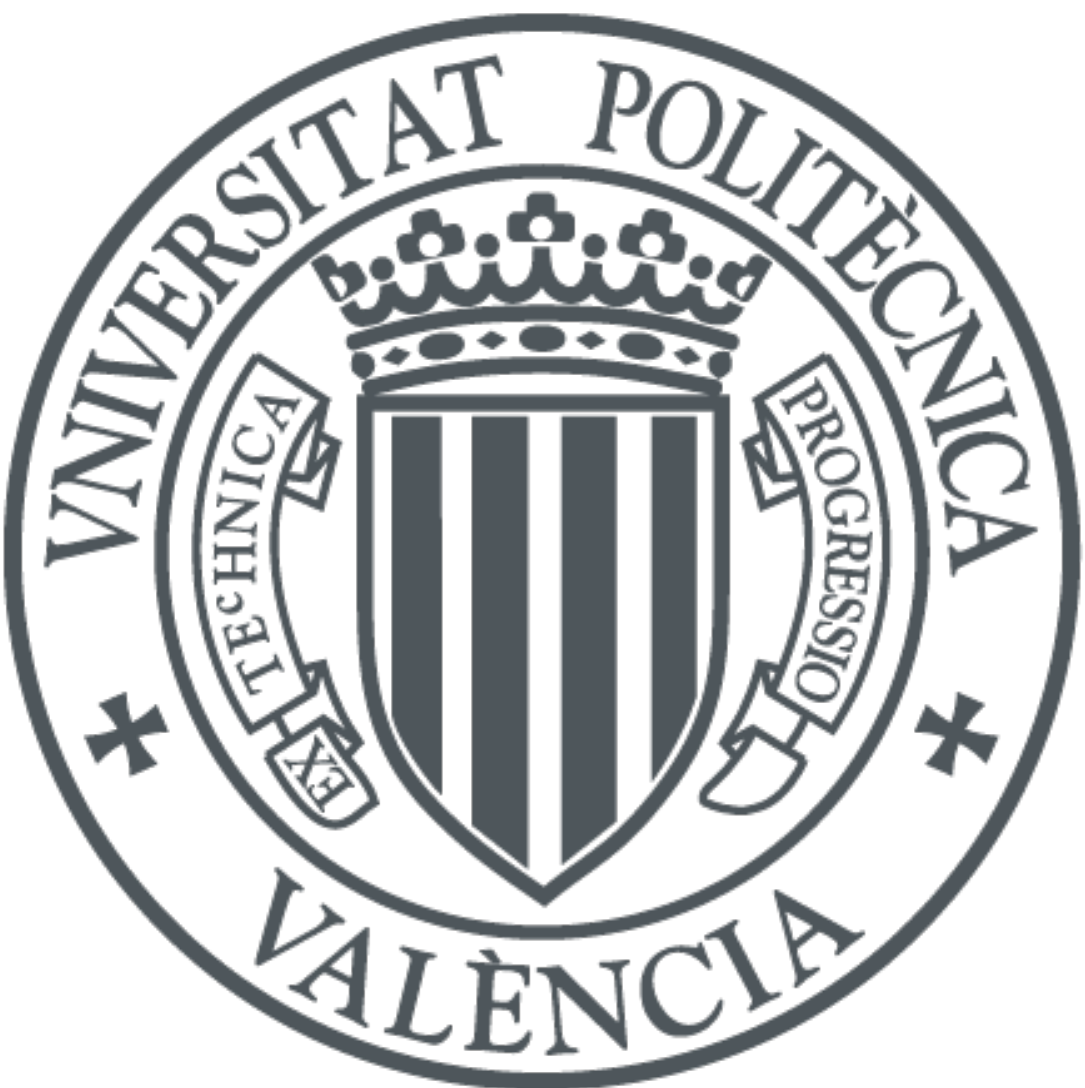

The final publication is available at

http://doi.org/10.1002/app.38118

Copyright John Wiley \& Sons

Additional Information 


\title{
Evaluation of Natural Rubber Specific Heat Capacity at high pressures from DSC experimental data at atmospheric pressure
}

\author{
P. Ortiz-Serna ${ }^{1 *}$, R. Díaz-Calleja ${ }^{1}$, M. J. Sanchis ${ }^{1}$ \\ ${ }^{1}$ Departamento de Termodinámica Aplicada, E.T.S.I.I., Instituto de Tecnología Eléctrica, \\ Universitat Politècnica de València, Camino de Vera s/n, 46022 Valencia, Spain. \\ *Corresponding author.e-mail: portiz@ter.upv.es
}

\begin{abstract}
The Natural Rubber (NR) specific heat capacity dependence on pressure was empirically determined from Differential Scanning Calorimetry (DSC) data, in the temperature range of -70 to $50{ }^{\circ} \mathrm{C}$, and by means of the Tait equation of state, in the pressure range of 0.1 to $240 \mathrm{MPa}$. It was found that the specific heat capacity decreases with pressure, being the dependency more pronounced at low pressures.
\end{abstract}

KEYWORDS. Natural Rubber, specific heat capacity, differential scanning calorimetry, high pressure.

\section{Introduction}

Heat capacity is a key magnitude of the Thermodynamic phase, because its entropy, enthalpy, etc., are derived from experimental data on isobaric heat capacity, $c_{p}(T, p$ $=$ constant ). Data are usually collected at atmospheric pressure. An important issue is to obtain the specific heat at higher pressures. Experimental data on heat capacity of condensed matter under high pressure are scarce and inaccurate, because a direct and accurate adiabatic calorimeter developed for ambient pressure can be hardly adapted to the devices generating 
high pressure [1-3]. This lack of experimental data is filled with model relationships, allowing one to derive heat capacity from the equation of state (EOS), interatomic potential, molecular dynamics, etc. Specifically, heat capacity increases with pressure, decreases, or remains almost insensitive with the pressure, depending on the model applied. It is difficult to recognize a general tendency in the changes of 'model' $c_{p}$ with pressure. For instance, heat capacity decreases with pressure for liquid $\mathrm{SiO}_{2}$ [4] and increases for liquid $\mathrm{MgO}$ [5]. It is reasonable that pressure increases frequency of atomic vibration in solids, increasing Debye temperature and, hence, decreasing heat capacity if the latter is less than the Dulong-Petit value $\left(3 R\right.$ per atom) $[6,7]$. The same is valid for organic solids and liquids, where $c_{p}$ decreases with pressure [8].

With regard to polymeric materials the information about the $c_{p}$ dependence on pressure is also scarce. For this reason it is convenient to elaborate a general strategy of calculation based on the classical thermodynamics to extend the knowledge of the specific heat from room to higher pressures. In this way, it is advisable to use an equation of state (EOS) as a model of behaviour in connection with the classical thermodynamic relations derived from the first and second laws of the thermodynamics. The purpose of the present paper is to propose a systematic calculation of the NR specific heat capacity [9] at higher pressures than the atmospheric one and in the same range of temperatures by means a specific Tait EOS.

\section{Experimental}

\section{1. Material}

Natural Rubber latex was obtained from Inducompre - Ind. Com. Prest. de Serviços Ltda. (Salvador, Brazil). Vulcanization additives used in the rubber formulation such as stearic acid (0.5 phr), zinc oxide (6 phr), sulphur (3.5 phr), N-t-butyl benzothiazole-2-sulfenamide 
(TBBS) (0.7 phr) and 4,4'-dioctyl-diphenylamine (1 phr) are present in the sample. The material was processed to obtain sheets with thickness of about $0.2 \mathrm{~mm}$.

\section{2. Experimental}

Differential Scanning Calorimetry (DSC).

DSC [10] thermogram was obtained for the NR sample at $10^{\circ} \mathrm{C} / \mathrm{min}$ in a DSC-Q10 equipment from TA Instruments. The gas used in the DSC-Q10 to obtain an inert atmosphere was $\mathrm{N}_{2}$.

Figure 1 illustrates the DSC thermogram obtained for the NR sample measured from 80 to $150^{\circ} \mathrm{C}$. In this Figure we can observe a sharp increase in the specific heat capacity as the NR is heated to a temperature above his glass transition temperature. The NR glass transition temperature $\left(T_{g}\right)$, evaluated as the temperature of the intersection of the glassyregion baseline with the tangent to the endotherm in the middle point, was of $-60.2^{\circ} \mathrm{C}$ [11]. It should be noted that strictly speaking DSC data are not taken in thermodynamic equilibrium in spite of the smallness of the sample.

\section{Thermodynamic basis}

One of the more relevant findings arising from the rational thermodynamics methodology [12] is that the laws of the Thermodynamics determine, at least partially, the constitutive structure of the material systems. This fact will be of usefulness in working on our problem. By considering a classical two-dimensional ideal system with space of state in a subset of the $(T, p)$-plane, one can define the differential forms of the work and heat as

$$
\begin{aligned}
& d w=v d p \\
& đ q=c_{p} d T+\lambda_{p} d p
\end{aligned}
$$


where $v(T, p)$ is the specific volume, $c_{p}(T, p)$ is the specific heat at constant pressure and $\lambda_{p}(T, p)$ is the latent heat with respect to the pressure. Then the first and second laws of Thermodynamics can be written as

$$
\begin{aligned}
& d h=c_{p} d T+\left(\lambda_{p}+v\right) d p \\
& d s=\frac{c_{p}}{T} d T+\frac{\lambda_{p}}{T} d p
\end{aligned}
$$

where $h$ and $s$ are the specific enthalpy and entropy, respectively.

Equivalently to eq. (2) one can write

$$
\begin{aligned}
& \frac{\partial c_{p}}{\partial p}=\frac{\partial \lambda_{p}}{\partial T}+\frac{\partial v}{\partial T} \\
& \frac{\partial c_{p}}{\partial p}=\frac{\partial \lambda_{p}}{\partial T}-\frac{\lambda_{p}}{T}
\end{aligned}
$$

These coupled differential equations allow us to obtain the coefficients of the heat differential form $\lambda_{p}$ and $c_{p}$ in terms of the coefficient of the work differential form. Once more, it is stressed that the important consequence of the eq. (3) is that the latent heat, $\lambda_{p}$, as well as the specific heat, $c_{p}$, can be predicted from the $\operatorname{EOS}[v=v(p, T)]$.

Solving eq. (3), the specific heat can be expressed in terms of the $p, v, T$ parameters appearing in the EOS as:

$$
c_{p}(T, p)=c_{p}{ }^{0}\left(T, p_{0}\right)-T \int_{p_{0}}^{p}\left(\frac{\partial^{2} v}{\partial T^{2}}\right)_{p} d p
$$

where $c_{p}{ }^{0}\left(T, p_{0}\right)$ is the value of the specific heat capacity at the reference pressure $p_{0}$ as a function of the temperature. Here $c_{p}{ }^{0}\left(T, p_{0}\right)$ will be taken from calorimetric data.

The calculus of the NR specific heat capacity at higher pressures was carried out starting from the $c_{p}{ }^{0}\left(T, p_{0}\right)$ values can be obtained by the Tait EOS [13] 


$$
v(p, t)=v\left(p_{0}, t\right)\left\{1-0.0894 \ln \left[1+\frac{p}{B(t)}\right]\right\}
$$

where $v\left(p_{0}, t\right)=\left(T_{a}+T_{b} t+T_{c} t^{2}\right)\left[\mathrm{cm}^{3} \cdot \mathrm{g}^{-1}\right]$ with $t$ in ${ }^{\circ} \mathrm{C}$, is the specific volume at atmospheric pressure, and $B(t)=\left(B_{a}\right) \exp \left(-B_{b} t\right)[\mathrm{MPa}]$ with $t$ in ${ }^{\circ} \mathrm{C}$, are the parameters in the melt state of NR.

In order to obtain the five parameters of the Tait EOS, standard literature data [14] of Pressure-Volume-Temperature $(p v T)$ for Natural Rubber were used. In Figure 2 are represented these NR $p v T$ data and their corresponding fit to the Tait EOS. The fit was carried out by means of a multiple nonlinear regression analysis of the literature data allowing the five characterizing parameters (i.e., $T_{a}, T_{b}, T_{c}, B_{a}, B_{b}$ ) to vary. The Tait parameters obtained in the fit were: $T_{a}=1.09023 \pm 0.00031 \mathrm{~cm}^{3} \mathrm{~g}^{-1}, T_{b}=5.6 \cdot 10^{-4} \pm 4 \cdot 10^{-6}$ $\mathrm{cm}^{3} \cdot \mathrm{g}^{-1} \cdot{ }^{\circ} \mathrm{C}^{-1}, T_{c}=9.72 \cdot 10^{-7} \pm 1.3 \cdot 10^{-8} \mathrm{~cm}^{3} \cdot \mathrm{g}^{-1} \cdot{ }^{\circ} \mathrm{C}^{-2}, B_{a}=218.77 \pm 1.43 \mathrm{MPa}$, and $B_{b}=4.98 \cdot 10^{-}$ ${ }^{3} \pm 3 \cdot 10^{-5} \mathrm{C}^{-1}$.

By means of eq. (5) and the obtained fit parameters, it should be possible to extrapolate the specific volume values of the polymer to higher pressures, as it is shown in Figure 2, where the isobaric curves from $0.1 \mathrm{MPa}$ to $240 \mathrm{MPa}$ are shown.

On the other hand, the specific heat capacity values, $c_{p}{ }^{0}\left(T, p_{0}\right)$, were obtained from DSC measurements by means of the following relationship

$$
c_{p}^{0}\left(T, p_{0}\right)=\frac{\dot{q}}{h} k
$$

where $c_{p}{ }^{0}\left(T, p_{0}\right)$ is in $\mathrm{J} \cdot \mathrm{g}^{-1} \cdot{ }^{\circ} \mathrm{C}^{-1}, \dot{q}$ is heat flow in $\mathrm{W} \cdot \mathrm{g}^{-1}, h$ is the heating rate in ${ }^{\circ} \mathrm{C} \cdot \mathrm{min}^{-1}$ (in our particular case was $10{ }^{\circ} \mathrm{C} \cdot \mathrm{min}^{-1}$ ), and $k=1.1007$ is the equipment calibration constant (dimensionless).

In Figure 3 is represented the temperature dependence of the specific heat capacity at atmospheric pressure, $c_{p}{ }^{0}(T, 0.1 \mathrm{MPa})$, from DSC measurements. As we can see, the $c_{p}{ }^{0}(T$, 
0.1 $\mathrm{MPa}$ ) increases with the temperature, showing a jump near to the glass transition as correspond to a second-order phase transition [9].

According to equation (4), and in order to obtain the specific heat capacity, $c_{p}(T, p)$, it is necessary to evaluate the first and second partial derivative of the volume with the temperature at constant pressure. These partial derivatives are obtained by using the Tait EOS and they are

$$
\begin{aligned}
& \left(\frac{\partial v}{\partial t}\right)_{p}=\left(T_{b}+2 \cdot T_{c} \cdot t\right)\left(1-N \cdot \ln \left(1+\frac{p}{B_{a} \cdot \exp \left(-B_{b} \cdot t\right)}\right)\right)- \\
& -\frac{\left(T_{a}+T_{b} \cdot t+T_{c} \cdot t^{2}\right) \cdot N \cdot p \cdot B_{b}}{B_{a} \cdot \exp \left(-B_{b} \cdot t\right) \cdot\left(1+\frac{p}{B_{a} \cdot \exp \left(-B_{b} \cdot t\right)}\right)} \\
& \left(\frac{\partial^{2} v}{\partial t^{2}}\right)_{p}=2 T_{c}\left[1-N \ln \left(1+\frac{p}{B_{a} \cdot e \exp \left(-B_{b} t\right)}\right)\right]-\frac{2\left(T_{b}+2 T_{c} t\right) N \cdot p \cdot B_{b}}{B_{a} \cdot \exp \left(-B_{b} t\right)\left(1+\frac{p}{B_{a} \cdot \exp \left(-B_{b} t\right)}\right)}- \\
& -\frac{\left(T_{a}+T_{b} t+T_{c} t^{2}\right) N \frac{B_{b}{ }^{2}}{p}+\frac{\left.T_{b} t+T_{c} t^{2}\right) N \cdot p^{2} \cdot B_{b}{ }^{2}}{B_{a}}}{B_{a} \exp \left(-B_{b} t\right)\left(1+\frac{B^{2}}{B_{a} \exp \left(-B_{b} t\right)}\right)} B_{a}^{2} \exp \left(-B_{b} t\right)^{2}\left(1+\frac{\exp \left(-B_{b} t\right)}{B_{a}}\right)^{2}
\end{aligned}
$$

where $N=0.0894$ and $t$ is the temperature in ${ }^{\circ} \mathrm{C}$.

After numerical integration [15] of eq. (4) and using the $c_{p}{ }^{0}(t, 0.1 \mathrm{MPa})$ values from the DSC measurements, specific heat capacity was calculated in the range of temperatures from 70 to $50{ }^{\circ} \mathrm{C}$ and in the pressure range from 0.1 to $240 \mathrm{MPa}$. The results are shown in Figure 4 . In this Figure we observe an increase of the specific heat capacity with temperature and a decrease with pressure, as the general tendency observed in solids [16].

In order to a clear visualization of the specific heat capacity dependence on temperature and pressure, we have plotted in Figures 5 and 6, respectively, the $c_{p}$ dependence on temperature for different isobars (from 0.1 to $240 \mathrm{MPa}$ with a $20 \mathrm{MPa}$ step) and the $c_{p}$ 
dependence on pressure for different isotherms, above and bellow the glass transition (from 70 to $50{ }^{\circ} \mathrm{C}$ with a $5^{\circ} \mathrm{C}$ step).

The same sharp increase of the specific heat capacity at temperatures near to the $T_{g}$ is also clearly visualized in Figure 6, in which we have plotted the specific heat capacity dependence on pressure at several temperatures. A first glance, it is observed a roughly linear specific heat capacity dependence on pressure, in the sense of a $c_{p}$ decrease with increasing pressure. However, a closed inspection by means of a zoom of one isotherm (see, for example the isotherm of $-40{ }^{\circ} \mathrm{C}$ in the top of the Figure 6) clearly shows that the $c_{p}$ dependence on pressure is more pronounced in the low pressure zone.

\section{Conclusions}

Specific heat capacity of the NR at pressures above the atmospheric one, was estimated from a thermodynamic expression that combines, on one hand, experimental $c_{p}$ data obtained by DSC and, on the other hand, $c_{p}$ data evaluated by means of the Tait EOS.

Our results are in accordance with those one reported for Andersson and Sundqvist [15] regarding to the relative specific heat capacity dependence on pressure for some polymers. Their results, in the same way as ours, show a nearly linearly decreasing of $c_{p}$ with pressure, with a more pronounced dependency at low pressures.

\section{Acknowledgements}

This work was financially supported by the DGCYT through Grant MAT2008-06725-C03 and by Generalitat Valenciana through Grant No. ACOMP/ 2010/204. 


\section{References}

[1] M. A. Kuznetsov, S. I. Lazarev, Meas. Tech. 48 (2005) 798.

[2] Y. Uwatoko, T. Fujiwara, M. Hedo, F. Tomioka, I. Umehara, J. Phys. Cond. Matt. 17 (2005) S1011.

[3] P. J. Van Ekeren, E. R. T. Bevers, J. Therm. Anal. Cal. 90 (2007) 931.

[4] B. B. Karki, D. Bhattarai, L. Stixrude, Phys. Rev. B 76 (2007) 104205.

[5] B. B. Karki, D. Bhattarai, L. Stixrude, Phys. Rev. B 732006174208.

[6] G. P. Johari, O. Andersson, Phys. Rev. B 73 (2006) 094202.

[7] I. Hamdi, M. Aouissi, A. Qteish, N. Meskini, Phys. Rev. B 73 (2006) 174114.

[8] Y. Miyake, D. Bessières, F. Plantier, H. Ushiki, C. Boned, J. Therm. Anal. Cal. 91 (2008) 347.

[9] J. W. Tester, M. Modell, Thermodynamics And Its Application, Chap. 8, Prentice Hall TTR, 1997.

[10] Michael E. Brown, Introduction to Thermal Analysis. Techniques and Applications, Kluwer Academic Publishers, 2004.

[11] P. Ortiz-Serna, R. Diaz-Calleja, M. J. Sanchis, G. Floudas, R.C. Nunes, A. F. Martins, L. L. Visconte, Macromolecules 43 (2010) 5094.

[12] C. Truesdell, Rational Thermodynamics, $2^{\text {nd }}$ ed. New York: Springer-Verlag, 1984.

[13] P.G. Tait, Physics and Chemistry of the Voyage of H. M. S. Challenger, Vol. 2, Part 4, HMSO London, 1888.

[14] P. Zoller, D. Walsh, Standard Pressure-Volume-Temperature Data for Polymers, Technomic Publ. Co., 1995.

[15] Matlab, The Language of Technical computing 7.0

[16] P. Andersson, B. Sundqvist, J. Polym. Sci. Polym. Phys. 13 (1975) 243. 


\section{Figure Captions}

Figure 1. DSC curve taken at $10^{\circ} \mathrm{C} \cdot \mathrm{min}^{-1}$ for NR.

Figure 2. Up: squares represent the NR pvt data (literature values), solid line is the fit to the Tait equation. Down: calculation of the specific volume values for other $p, t$ conditions by means of the obtained fit.

Figure 3. Specific heat capacity as a function of temperature, at atmospheric pressure, for NR.

Figure 4. Specific heat capacity as a function of temperature and pressure for NR.

Figure 5. Temperature dependence of the isobaric specific heat capacity, $c_{p}\left(\mathrm{~J} \cdot \mathrm{g}^{-1} \cdot{ }^{\circ} \mathrm{C}^{-1}\right)$ (from 0.1MPato 240MPa with a step of 20MPa).

Figure 6. Pressure dependence of the isothermal specific heat capacity, $c_{p}\left(\mathrm{~J} \cdot \mathrm{g}^{-1} \cdot{ }^{\circ} \mathrm{C}^{-1}\right)$ (from 70 to $50^{\circ} \mathrm{C}$ with a step of $5^{\circ} \mathrm{C}$ ). 


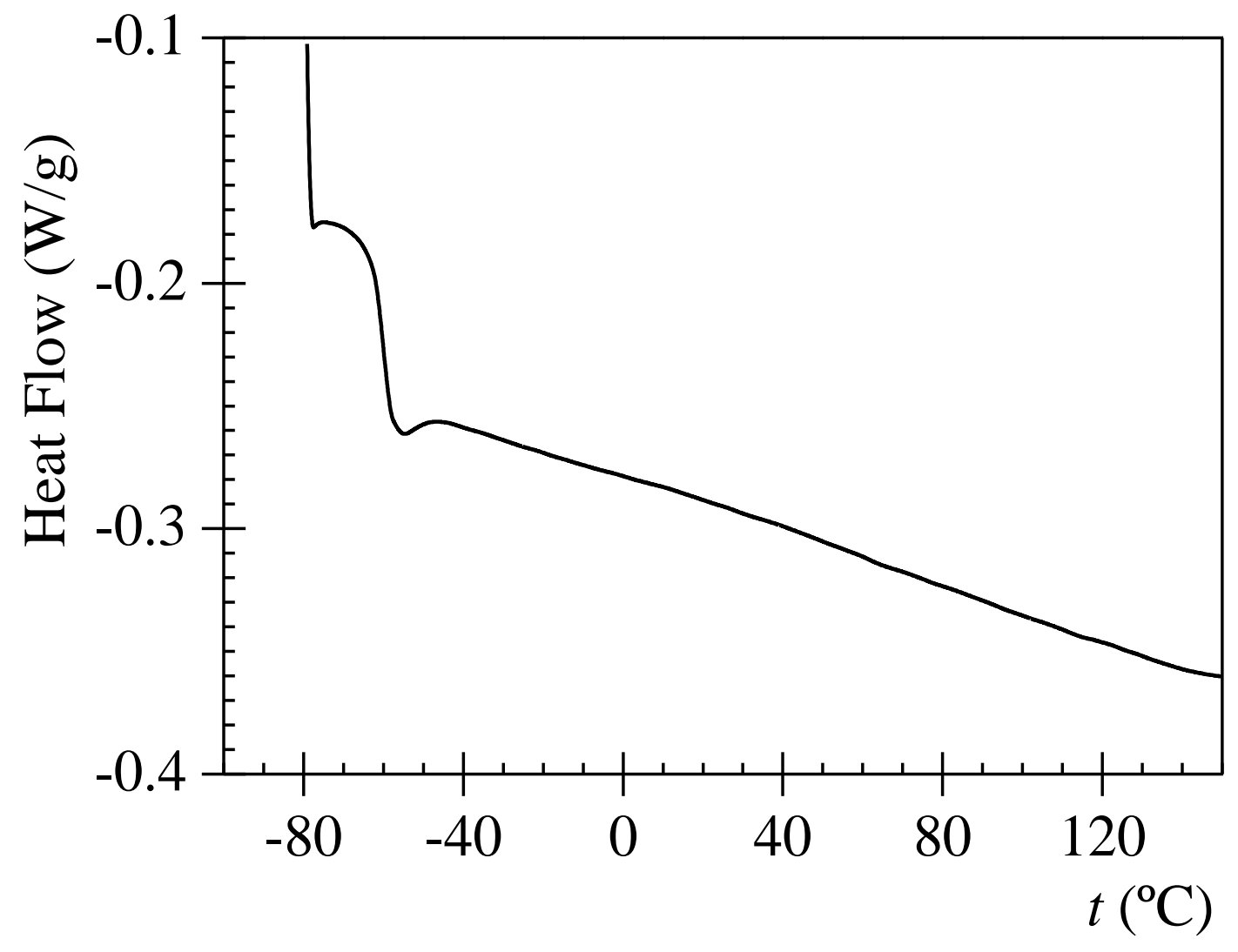

Figure 1. 


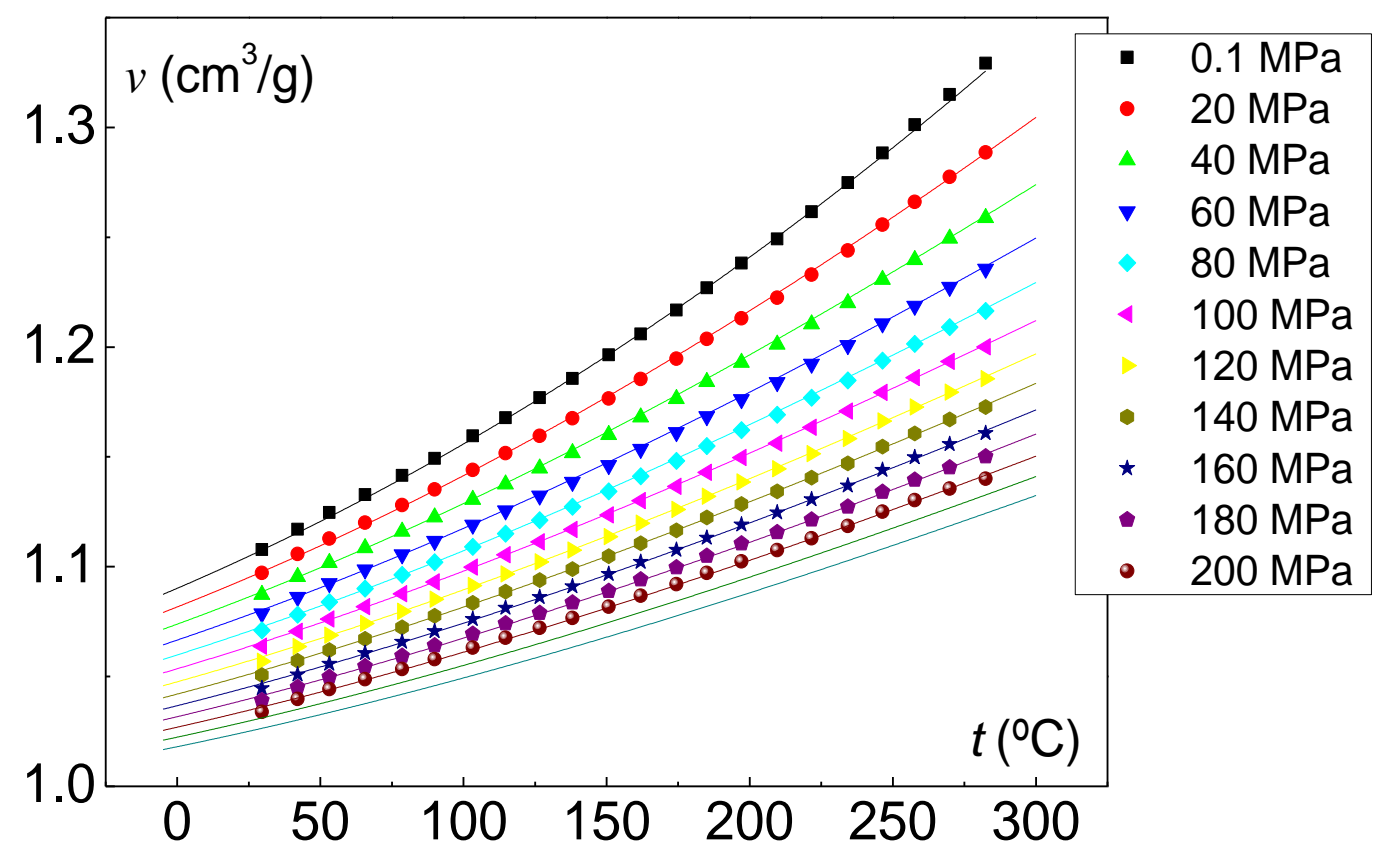

Figure 2. 


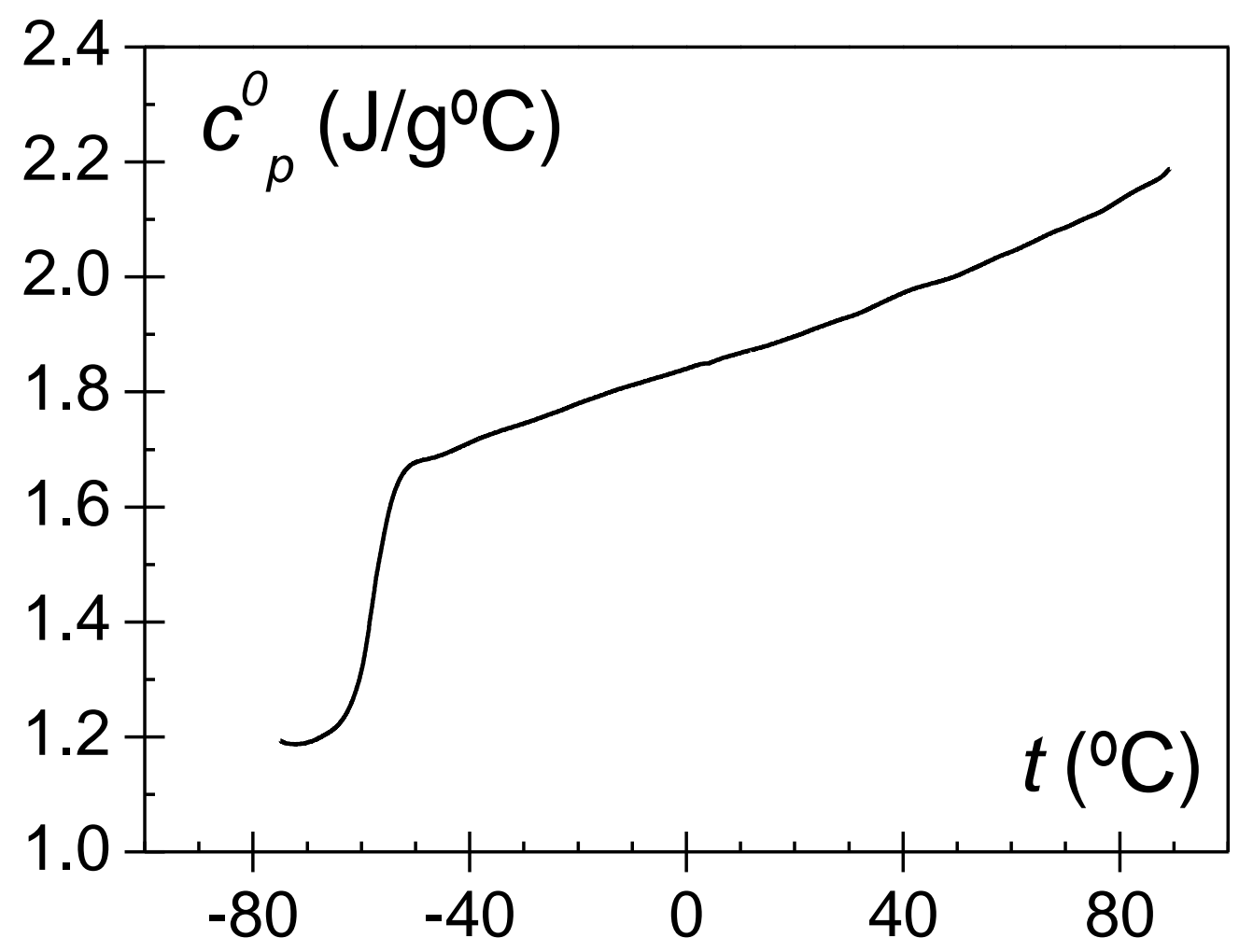

Figure 3. 


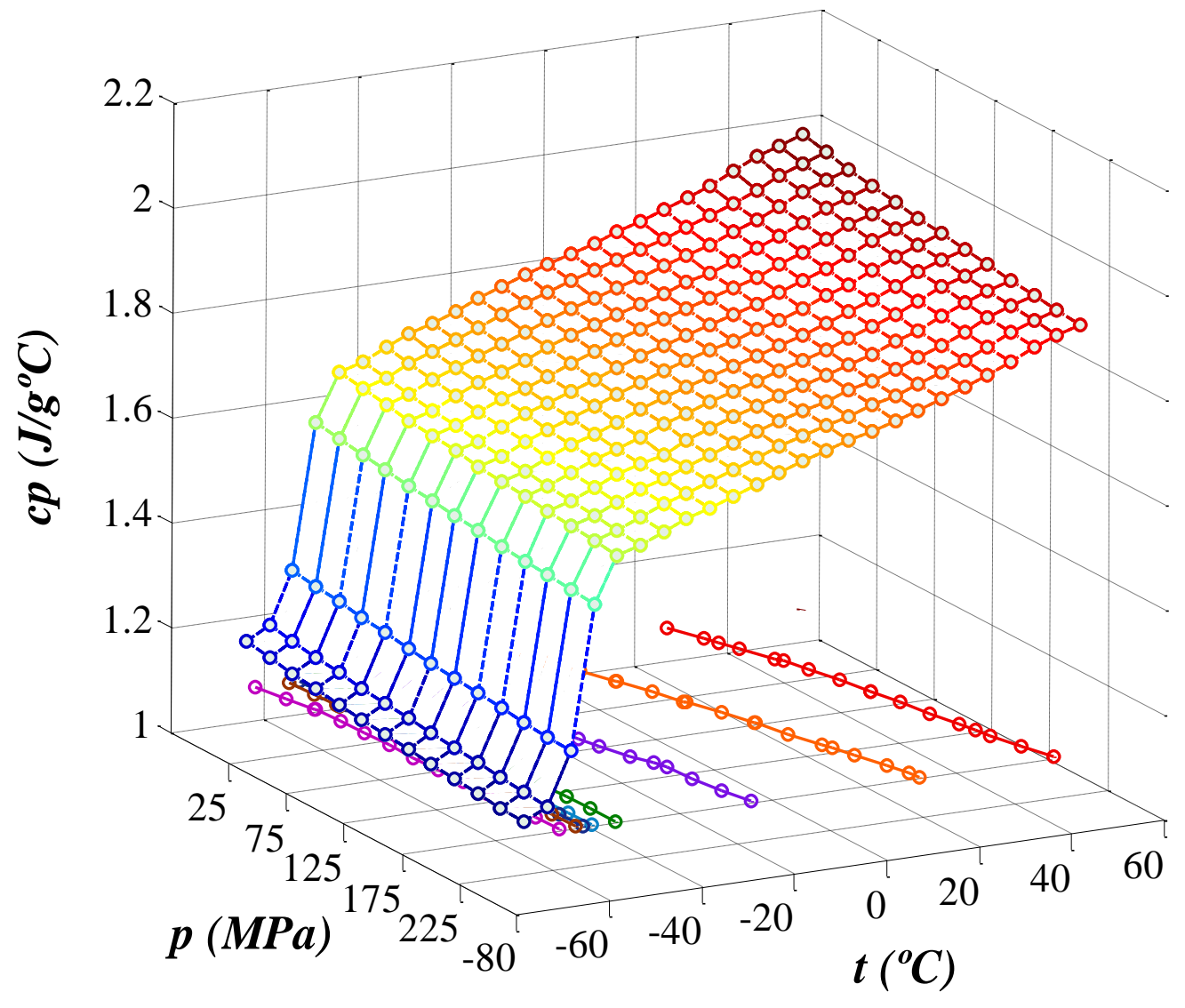

Figure 4. 


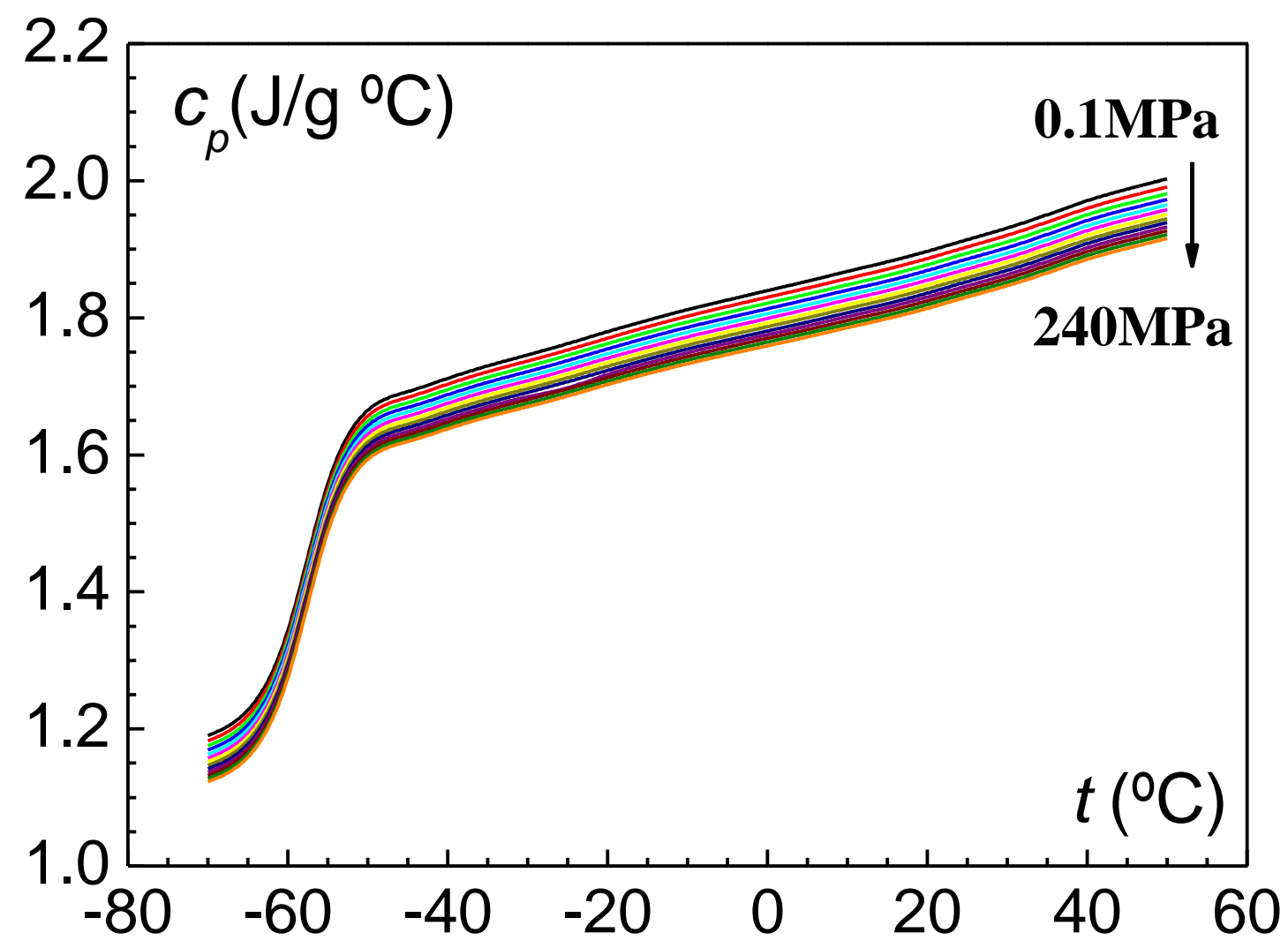

Figure 5. 


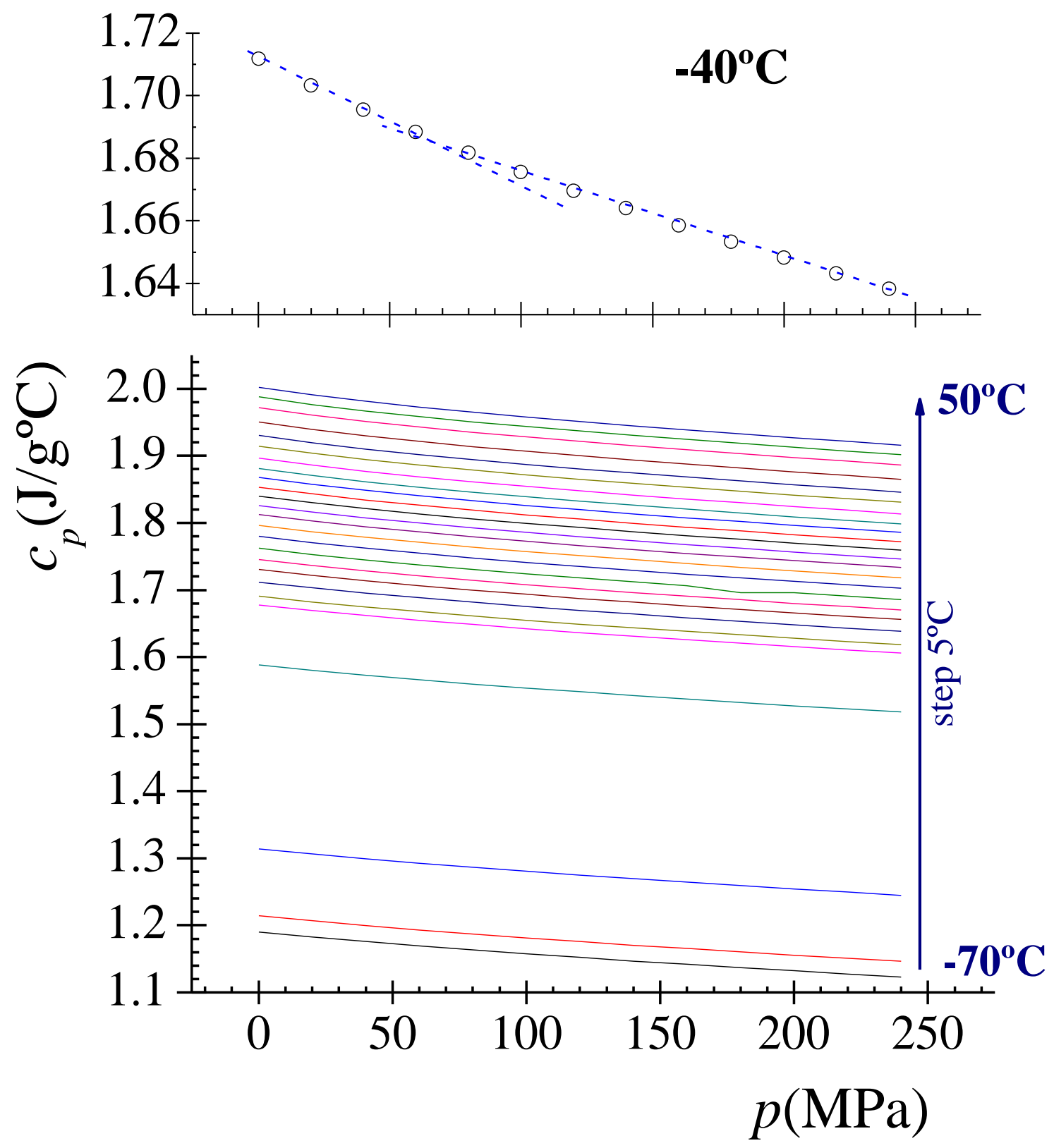

Figure 6. 